\title{
Null EPAC mutants reveal a sequential order of versatile cAMP effects during Drosophila aversive odor learning
}

\author{
Antje Richlitzki, Philipp Latour, and Martin Schwärzel \\ Freie Universität Berlin, Biology/Neurobiology, D-14195 Berlin, Germany
}

\begin{abstract}
Here, we define a role of the CAMP intermediate EPAC in Drosophila aversive odor learning by means of null epac mutants. Complementation analysis revealed that EPAC acts downstream from the rutabaga adenylyl cyclase and in parallel to protein kinase A. By means of targeted knockdown and genetic rescue we identified mushroom body Kenyon cells (KCs) as a necessary and sufficient site of EPAC action. We provide mechanistic insights by analyzing acquisition dynamics and using the "performance increment" as a means to access the trial-based sequential organization of odor learning. Thereby we show that versatile cAMP-dependent mechanisms are engaged within a sequential order that correlate to individual trials of the training session.
\end{abstract}

The cAMP signaling pathway is central to the regulation of plasticity and can mediate cellular responses via different intermediaries, i.e., PKA (protein kinase A), EPACs (exchange proteins directly activated by cAMP), and CNGs (cyclic nucleotide gated channels) (Laurent et al. 2012; Schmidt et al. 2013; Cooper and Tabbasum 2014; He et al. 2014). While the numerous contributions of PKA to the regulation of plasticity have been described in great detail (Kandel 2001), the role of EPAC was not recognized until 1998 (de Rooij et al. 1998; Kawasaki et al. 1998). Since then, its operation as a noncanonical cAMP sensor has been proven in numerous studies, aided by the development of selective cAMP analogs and/or genetic models that allow discrimination between PKA and EPAC functions (Enserink et al. 2002; Bos 2006; Bertinetti et al. 2009; Gloerich and Bos 2010; Efetova et al. 2013). Epac has been shown to enhance neurotransmitter release (Sakaba and Neher 2003; Zhong and Zucker 2005), activate neuronal excitability via $\mathrm{Ca}^{2+}$-dependent $\mathrm{K}^{+}$-channels (Ster et al. 2007), and enhance hippocampal long-term potentiation and memory consolidation (Gelinas et al. 2008; Ma et al. 2009; Yang et al. 2012). Here, we investigated a potential role of Epac in the Drosophila aversive odor-learning paradigm.

\section{Results}

Deletions at the unique Drosophila epac locus were generated by remobilizing FRT-containing P-elements partially covering the epac locus (Fig. 1A): epac ${ }^{\Delta 1}$ was generated by combining d04690 and f07038, epac ${ }^{\Delta 2}$ by combining e00785 and f07038, and epac $^{\Delta 3}$ by combining e00785 and f00899. Deletions were homozygous lethal but viable offspring were obtained for transcombinations of $\Delta 1 / \Delta 2$ and $\Delta 1 / \Delta 3$ that covered a large part of the locus and effectively prohibited epac transcription (Efetova et al. 2013). Compared with wild-type strains, null epac mutants showed reduced performance in an aversive associative odor learning task $\left(F_{(2,31)}=28.16, P<0.01\right)$ while odor perception $\left(F_{(2,36)}=2.87, P=0.47\right.$ and $\left.F_{(2,34)}=0.23, P=<0.80\right)$ and shock reactivity $\left(F_{(2,32)}=0.08, P=0.93\right)$ were unaffected (Fig. 1B). Together, these results suggest that learning defects seen in null epac mutants were not simply due to impaired stimulus percep-

Corresponding author: martin.schwaerzel@fu-berlin.de Article is online at http://www.learnmem.org/cgi/doi/10.1101//m.043646.116. tion but rather that EPAC mediates specific aspects of cAMP signaling during aversive odor learning.

\section{Epac supports STM at the level of Kenyon cells}

In search of the neuronal circuits of the fly brain where EPAC supports odor learning we applied a dual strategy designed, first, to identify necessary structures by means of RNAi knockdown, and second, to test their sufficiency by means of genetic rescue. We proved this concept by using the pan-neuronal elav-Gal4 driver that effectively phenocopied null epac mutant performance when combined with the RNAi-knockdown construct, and restored aversive odor learning upon expressing an $\mathrm{epac}^{+}$ cDNA within an otherwise null epac mutant brain (Fig. 2A; $\left.F_{(6,60)}=15.56, P<0.001\right)$. Genetic controls that bore either the elav-Gal4 driver or UAS-epac transgenes alone did not impact on performance of appropriate genetic backgrounds ( $P S>0.05)$.

Next, we restricted epac knockdown to the majority of Drosophila mushroom body Kenyon cells (KCs), a recognized Drosophila "learning center" (Heisenberg 2003), by use of mb247-Gal4. Similar to results of pan-neuronal manipulation we observed reduced odor learning with the knockdown approach while genetic rescue restored learning to wild-type levels $\left(F_{(6,64)}=15.95, P<0.001\right.$; Fig. $\left.2 \mathrm{~B}\right)$. Altogether, these results identify KCs as a necessary and sufficient element of EPAC function in support of odor learning.

Finally, we aimed to distinguish between acute functions of EPAC at the adult stage or alternatively during development by use of the TARGET system (McGuire et al. 2003). To that end we induced expression of double-stranded RNAi (Fig. 2C) or rescuing transgene (Fig. 2D) at the adult stage. In line with an acute function during the learning act, induced knockdown of EPAC diminished learning $\left(F_{(5,45)}=5.29, P<0.001\right.$; Fig. $\left.2 \mathrm{C}\right)$ while, conversely, its induced rescue elevated performance to wild-type levels $\left(F_{(5,47)}=35.64, P<0.001\right.$; Fig. 2D). Genetic controls that bore either the mb247-Gal4 driver or appropriate epac transgenes

\footnotetext{
(C) 2017 Richlitzki et al. This article is distributed exclusively by Cold Spring Harbor Laboratory Press for the first 12 months after the full-issue publication date (see http://learnmem.cshlp.org/site/misc/terms.xhtml). After 12 months, it is available under a Creative Commons License (AttributionNonCommercial 4.0 International), as described at http://creativecommons. org/licenses/by-nc/4.0/.
} 

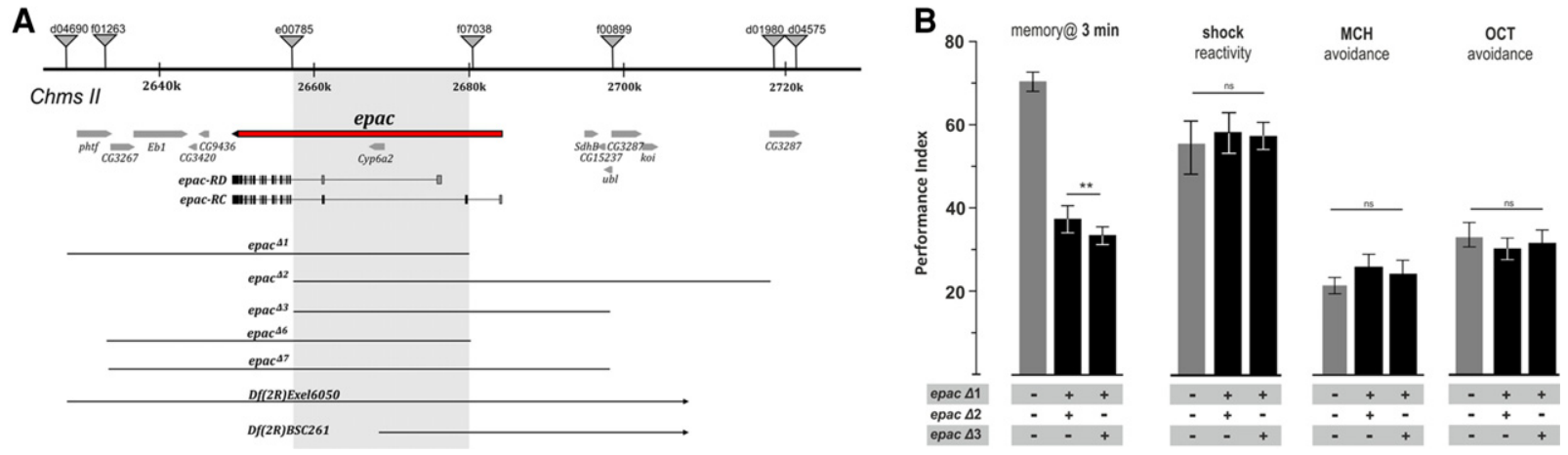

Figure 1. Null epac mutants show impaired short-term memory. Trans-combinations of small deletions generate null epac mutants that exhibit normal sensory acuity but impaired aversive odor learning. $(A)$ Deletion of the epac locus was achieved by remobilizing FRT-containing P-elements; epacD1 by combining d04690 // f07038, epacD2 by combining e00785 // xxx, and epacD3 by combining e00785 // f00899. Trans-combinations of D1/D2 and D1/D3 removed the coding sequence of the unique Drosophila epac locus. (B) Loss of EPAC reduced performance of short-term memory (STM) measured 3 min after a single cycle training session while shock reactivity and odor avoidance remained unchanged. All data represent means \pm SEM. $N=6-8$ for behavioral results. Statistical differences at the level of $P \leq 0.01$ are denoted by asterisks.

alone did not impact on performance, neither did temperature (Ps > 0.05).

\section{EPAC and PKA act in a similar pathway downstream from rutabaga}

How do these new findings regarding epac connect to previously known aspects of cAMP signaling underlying Drosophila aversive STM? Particularly, the reduction of performance from wild-type levels to $\sim 50 \%$ residual learning seen in null epac mutants suggests engagement of alternative downstream mechanisms. Therefore, we tested for a contribution of protein kinase A (PKA) using double-stranded RNAi directed against DC0, the most abundant PKA catalytic subunit in fruit flies (Lane and Kalderon 1993).

Pan-neuronal knockdown of DC0 effectively decreased PKA activity as determined in brain homogenates $\left(F_{(2,32)}=25.32\right.$, $P<0.01$; Fig. $3 \mathrm{~A})$ and significantly reduced aversive odor learning $\left(F_{(3,32)}=15.46, P<0.01\right.$; Fig. $\left.3 \mathrm{~B}\right)$. In contrast, shock reactivity and odor acuity remained on wild-type levels formally excluding impaired perception of task relevant stimuli as a potential cause for learning impairment (Ps $>0.05$; Fig. 3C). Next, we tested whether PKA might play a role in KCs by means of mb247 using the TAGET system to induce knockdown at the adult stage. Thereby, we showed an acute role of PKA during the learning act rather than a developmental contribution $\left(F_{(7,73)}=11.99\right.$, $P<0.001$; Fig. 3D). Moreover, these data revealed that PKA and EPAC act at the same stage of the learning-relevant circuit, i.e., mb247-marked KCs.

Next, we addressed their functional relationship by means of double manipulation showing that animals that bore either the epac mutation, the PKA knockdown, or the knockdown in an epac mutant background all performed indistinguishably from one another on reduced levels $\left(F_{(4,47)}=23.93, P<0.001\right.$; Fig. $3 \mathrm{E})$. These data suggest that PKA and EPAC support aversive odor learning via a common pathway. Moreover, the two cAMP intermediaries do so downstream from the $\mathrm{Ca}^{2+}$ sensitive type I adenylyl cyclase rutabaga (rut-AC1), as performance of rut1 does not interact with loss of either epac or PKA $\left(F_{(5,57)}=1.22, P=\right.$ 0.32; Fig. 3F).

\section{EPAC designates a particular fraction of rut-dependent STM}

After fixing the position of EPAC within biochemical pathways and neuroanatomical circuits underlying aversive odor learning, we aimed to gain mechanistic insights into its mode of action by analyzing the acquisition dynamics (Moressis 2009 \#6912). To that end we varied the number of trials experienced during a single-cycle training session as indicated (Fig. 4A) and assayed performance in wild-type, null epac, and rut ${ }^{1}$ mutant flies. For comparison, we included a wild-type group trained at low US saliency, i.e., with $15 \mathrm{~V}$ DC instead of the $120 \mathrm{~V}$ DC high saliency conditions normally used. While all highly salient trained groups performed at similar levels after one-trial training $\left(F_{(2,27)}=0.21, P=0.81\right)$, wild-type dissociated from mutants after two-trial training $\left(F_{(2,33)}=13.31, P=0.001\right)$ while thereafter all genotypes were completely ungrouped $\left(F_{(2,36)}=13.38, P=0.03\right)$.

In order to access dynamic aspects of this dissociation over the time course of training we performed an alternative evaluation and calculated the "performance increment" of individual trials as the difference between the mean PI values of consecutive trials. The error bars (SEMs) of performance increments were determined by adding the variances $\left(\sigma^{2}\right)$ of single PI values of appropriate trials according to the law of error propagation, i.e., $\mathrm{SEM}=$ $\sqrt{ }\left\{1 / N \times\left(\sigma^{2}(\operatorname{trial} x)+\sigma^{2}(\operatorname{trial} x+1)\right)\right\}$. Thereby we found that during the first trial all genotypes learned to a similar extent (Fig. 4C). Statistical analysis was performed using a two-way ANOVA showing significant effects of trial $\left(F_{(2,59)}=45.35, P<0.001\right)$ but not of genotype $\left(F_{(2,55)}=0.68, P=0.51\right)$ nor their interaction $\left(F_{(3,59)}=\right.$ $0.51, P=0.61)$. During the second trial only the wild-type $(+/+)$ acquired new information in contrast to null epac and rut $^{1}$ mutants, which retained performance at previous levels without further increment (ANOVAs for trial: $F_{(2,78)}=5.12, P<0.05$; genotype: $F_{(3,75)}=19.2, P<0.05$; interaction: $F_{(3,75)}=1.15, P=$ 0.21 ). However, from the third trial onward the situation changed when the two mutants dissociated; while rut ${ }^{1}$ mutants failed to acquire new information, null epac flies showed wild-type levels of learning (Fig. 4E; ANOVAs for trial: $F_{(2,64)}=27.64, P<0.01$; genotype: $F_{(3,64)}=8.62, P<0.01$; interaction: $F_{(3,64)}=5.36, P<$ 0.01 ). Moreover, epac mutants asymptote a stable plateau by 12 trials (Fig. 4F), as did the wild-type, although at a reduced level (compare 12 to 24 trials in Fig. 4B).

Thereby, our analysis of the performance increment revealed the true nature of the learning impairment seen in epac mutants, i.e., the lack of performance fortification by $\sim 15 \%$ that was triggered during the second training trial. In contrast, acquisition of epac-independent STM was normal in epac mutants as illustrated by parallel slopes of the learning curve of wildtype and mutants under highly salient training conditions, i.e., $120 \mathrm{~V}$ DC (Fig. 4B). 
A
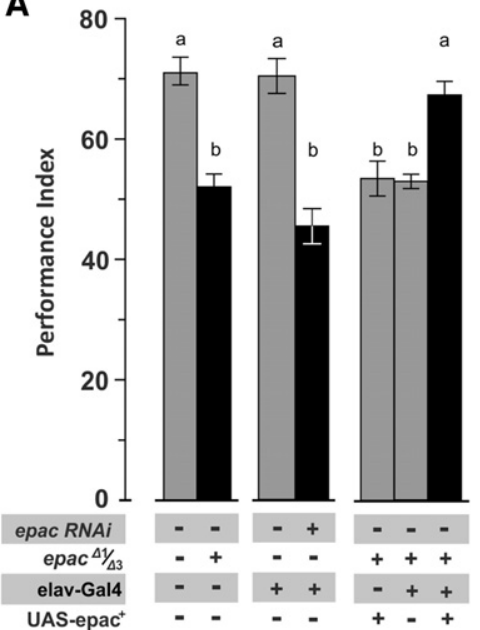

B

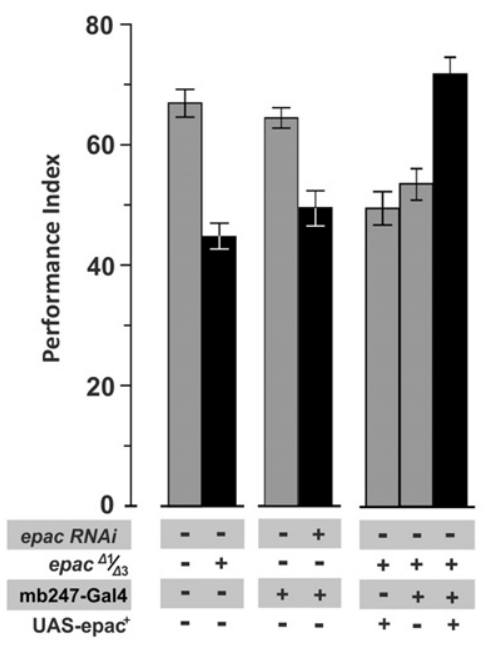

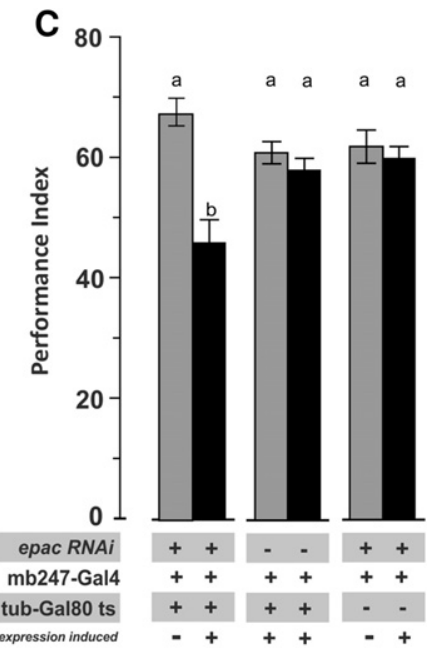

D

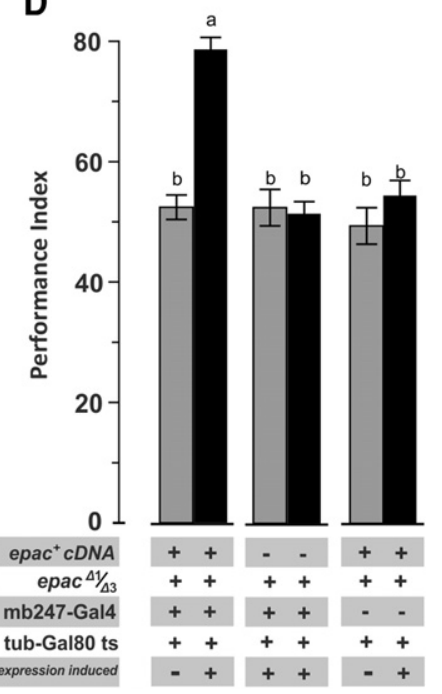

Figure 2. Epac supports STM formation at the level of the mushroom body Kenyon cells. Genetic manipulation of EPAC function determines STM performance. $(A)$ Pan-neuronal expression of an epacspecific RNAi transgene mimics performance of null epac mutants, while pan-neuronal rescue restores performance to wild-type levels. $(B)$ Restricting expression of transgenes to the mushroom body by means of mb247-Gal4 identifies Kenyon cells as a necessary and sufficient section of EPAC function to support STM. (C) Induced EPAC knockdown at the adult stage by means of the TARGET system excludes developmental contributions to loss of STM. (D) Induced EPAC rescue at the adult stage restores STM performance. All data represent means \pm SEM. $N=6-8$. Statistical differences at the level of $P \leq$ 0.05 are denoted by different letters.

\section{Discussion}

Here, we distinguish epac-dependent from epac-independent learning by means of a Drosophila null-epac mutant and use the performance increment as a means to address the functional disparity of individual training trials. Disparity impacts on the learning rate suggesting an evolutionary benefit of alternative cAMP mediators as this provides a mechanism for transforming rutderived cAMP signals into behavioral output following different strategies.

\section{Parallel processing within the $\mathrm{KC}$ matrix via disparate synaptic hysteresis}

Rutabaga adenylyl cyclase (rut-AC1) is supposed to act as coincidence detector between US- and CS-derived impulses that con- verge at the level of $\mathrm{KC}$ synapses and consequently induce cAMP-dependent plasticity within an odor specific matrix of KCs. This KC synapse matrix is widely accepted as a neuronal representation of learning and thought of as an engram of an odor memory (Heisenberg 2003). Dopaminergic neurons (DANs) provide the major share of KCs' dopamine-induced cAMP signals, however KC synapses are not uniform but exhibit different sensitivities for cAMP (Aso et al. 2010; Boto et al. 2014). Likewise, learning is not an instantaneous process but develops over the multiple trials of a training session. As a consequence, cAMP gain over the time course of training would not be uniform but effectively determined by the sensitivity of a particular KC synapse within the odor specific matrix. This disparity would proceed to the next levels, i.e., activation gain of alternative downstream cAMP intermediaries that, in turn, are unequally sensitive as reflected by characteristic half-maximal cAMP concentrations $\left(\mathrm{IC}_{50}\right)$, i.e., $\mathrm{IC}_{50}$ values for $\mathrm{PKA} \approx 80 \mathrm{~nm}$; for $\mathrm{HCN}$ channels $\approx 100 \mathrm{~nm}$; and for $\mathrm{EPAC} \approx 800$ $\mathrm{nm}$ (Rocher et al. 2009; Chen et al. 2013). By this design, KCs diversify into high and low cAMP gain fractions that activate mediators responsive to either high and/or low cAMP amplitudes at quite different rates. Thereby, a particular odorspecific KC matrix can interconnect to multiple outputs via different mechanisms of cAMP-dependent plasticity as its synaptic elements exhibit disparate hysteresis, i.e., different time-dependent changes in the cellular cAMP levels.

\section{Disparate processing generates alternative learning strategies}

Our surprising result that insensitive EPAC recruits early, i.e., during the second trial of the training session, suggests existence of a fast, high-amplitude cAMP signal that meets EPAC's thresholds early during training. Similarly, one has to assume that the efficacy of later training trials is mediated by a perpetuating low-amplitude signal that develops with low gain over multiple trials. Given the fact that PKA acts isogenic to EPAC and null epac mutants show regular learning during late training trials-that is from the third trial onwardneither of these intermediates is likely to account for late trial learning. One plausible mechanism would be HCN channels, i.e., subthreshold, voltage-gated ion channels that reduce membrane resistance and promote neuronal firing probability (Benarroch 2013). Within sparsely firing KCs such channels might stabilize an odor-specific synaptic matrix, i.e., the CS-representation, over the 1-min time course of the training cycle and promote its transfer to mushroom body output neurons (MBONs), the recognized convergence site for KCs (Hige et al. 2015; Owald and Waddell 2015; Barnstedt et al. 2016). In contrast, epac has been shown to enhance neurotransmitter release (Sakaba and Neher 

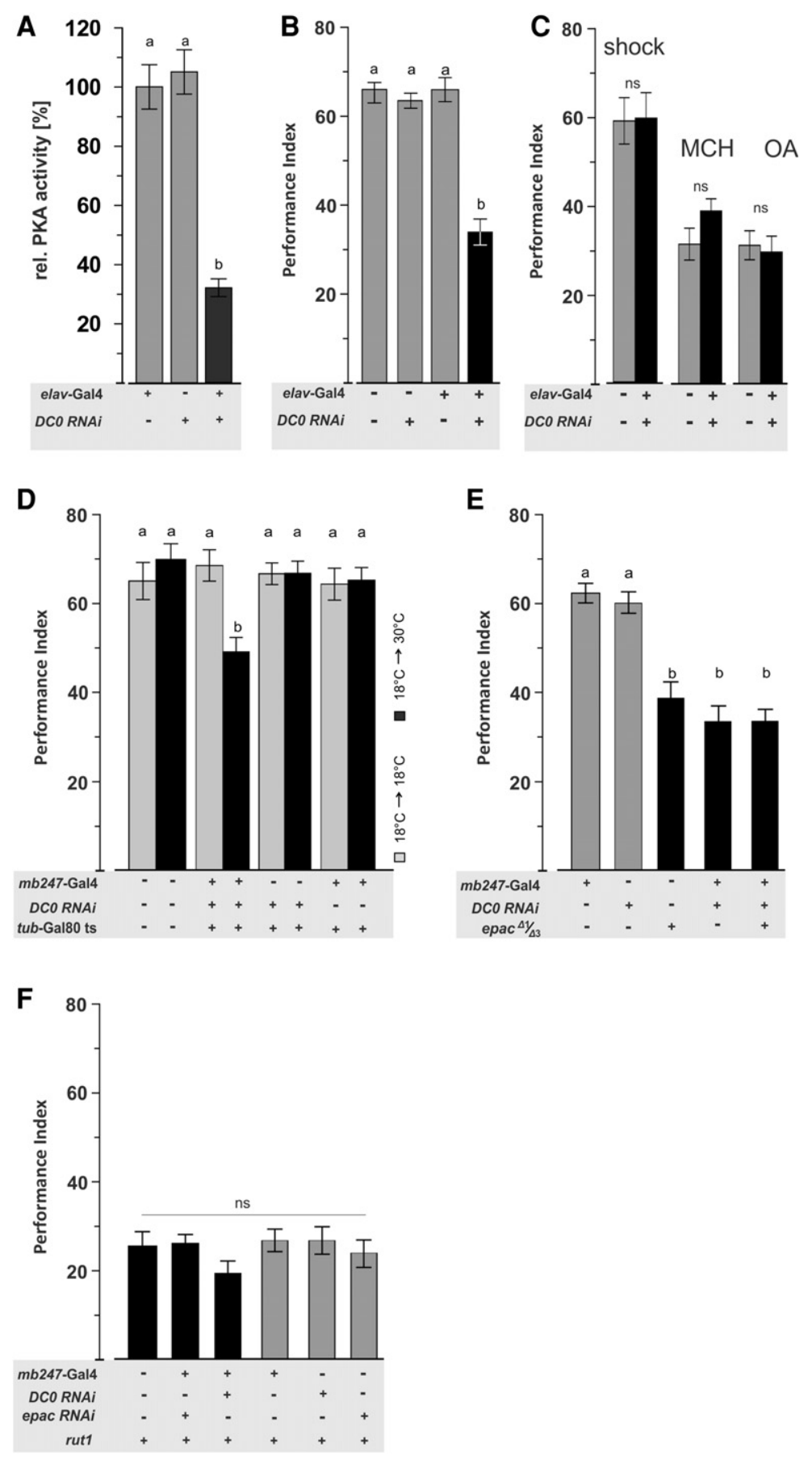

Figure 3. EPAC and PKA act via a similar pathway downstream from rutabaga. STM performance is not sensitive to genetic interactions between the CAMP intermediaries PKA and epac with each, other or with the rutabaga adenylyl cyclase. $(A)$ Knockdown of $D C 0$, Drosophilás most abundant catalytic PKA subunit reduced PKA activity of brain homogenate. (B) Pan-neuronal DC0 knockdown diminishes STM performance. $(C)$ Shock reactivity and odor acuity are not affected by DC0 knockdown. $(D)$ Induced knockdown of $D C O$ at the adult stage by means of the TARGET system diminishes STM performance. $(E)$ STM is not sensitive to interactions between the CAMP intermediaries PKA and epac. ( $F$ ) STM is not sensitive to interactions between the rutabaga adenylyl cyclase and PKA or EPAC. All data represent means \pm SEM. $N=6-8$. Statistical differences at the level of $P \leq 0.05$ are denoted by different letters.

2003; Zhong and Zucker 2005) and/or activated neuronal excitability (Ster et al. 2007) via different mechanisms.

While epac-dependent and independent learning mechanisms clearly dissociate at the molecular level, their learning rates appear counter-intuitive as a highamplitude signal precedes a low-amplitude one, but both originate from rut-AC1. If one considers the animals need to trade off certainty of a prediction against its computation time, this seemingly counterintuitive design appears deliberate and beneficial as it holds the possibility of combining both contrarian needs (DasGupta et al. 2014): First, epacdependent learning requires a short computation time, i.e., after two trials, compared with the slow and cumbersome integration over multiple trials needed for epac-independent learning. Second, epac-dependent learning is restricted to salient conditions, i.e., high voltages that represent a serious threat to the animal's health, while this part of the animal's memory is spared with the $15 \mathrm{~V}$ DC US (see Fig. 4B). In fact, wild-type strains trained with $15 \mathrm{~V}$ exhibited similar learning to epac-null mutants trained with $120 \mathrm{~V}$ suggesting that epac amplifies conditioned avoidance under trusted environmental circumstances.

\section{Do training trials clock recurrent computation within the learning network?}

How individual training trials are represented within the fly brain is unclear. However, functional studies have identified DANs as critical substrates of the US that tightly innervate KCs at the level of the MBs. In general, DANs and KCs work together with MBONs, the recognized readout routes of aversive odor memory (Bouzaiane et al. 2015). Moreover, their anatomy suggests that MBONs serve as critical inter-loops that reiterate the MBs' computational output to DANs, i.e., its major modulatory input: This recurrent connectivity exhibits a remarkable zonal architecture as dendrites of MBONs tile the length of KC axons in a nonoverlapping manner, where they meet with dopaminergic neurons (DANs), the other main innervation of the MB lobes. The DANs tile the MB lobes in a corresponding manner so that the dendrites of a particular MBON meet axonal projections of cognate DANs. Moreover, dendrites of DANs overlap with MBON axons within the MBON projection zones outside the MBs suggesting that MBONs modulate the activity of DANs and thereby generate recurrent loops (Aso et al. 2014). By this design MBON activity is dually modulated by DANs, first via a direct connection, and second via a KC detour that undergoes cAMP-dependent plasticity. However, further research will be required to understand the rules by which repetitive trials clock the computation within the DAN/KC/MBON network. 
A

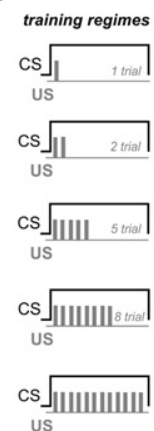

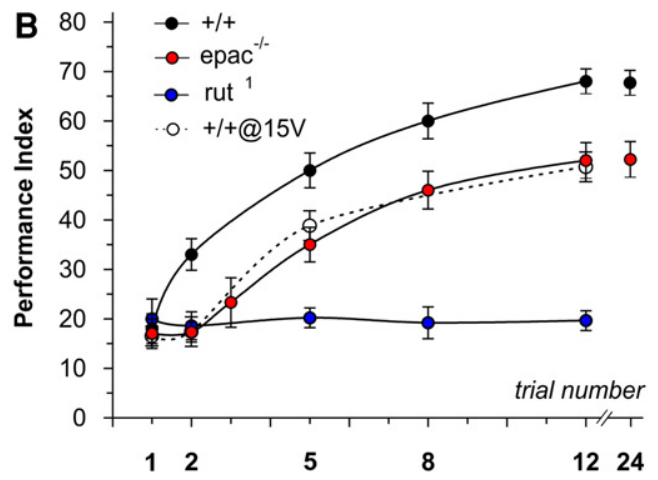

C

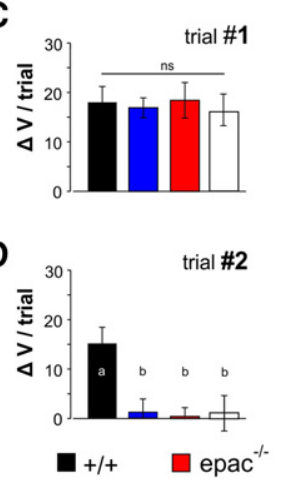

$\mathbf{E}$

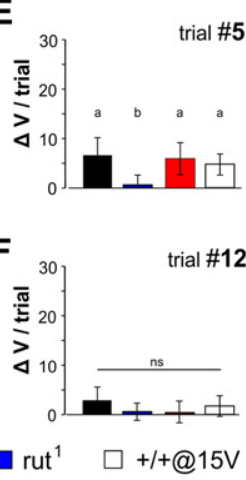

Figure 4. Null epac mutants exhibit altered acquisition during single-cycle training. During single-cycle training animals receive a variable number of shock trials - the unconditioned stimulus (US)_in the presence of an olfactory cue, the conditioned stimulus (CS). ( $A$ ) Scheme of single-cycle training conditions that provide ascending training trials during a fixed period of CS presentation. (B) To determine the contribution of individual trials we varied the number of trials received during training and plotted performance as a function of trial number for wild-type, rut ${ }^{1}$ and null epac mutants. We calculated the amount of newly acquired information normalized per trial for $(C)$ first trial, $(D)$ second trial, $(E)$ fifth trial, and $(F)$ 12th trial. All data represent means \pm SEM. $N=6-8$. Statistical differences at the level of $P \leq 0.05$ are denoted by different letters.

\section{Materials and Methods}

\section{Fly care}

Flies were raised at $24^{\circ} \mathrm{C}$ and $60 \%$ relative humidity with a $14: 10 \mathrm{~h}$ light-dark cycle on cornmeal-based food following the Würzburg recipe (Guo et al. 1996). Genetic crosses were performed according to standard procedures. All experiments were performed with 3- to 5-d-old male F1 progeny of homozygous parental lines. All lines used in this study were outcrossed to Canton-S. As a result of outcrossing null epac mutants' performance shifted from $<40$ PI in the original, unspecified genetic background (see Fig. 1B) to $>50$ PI after outcrossing to Canton-S for more than five generations (see Figs. 2D, 4A).

\section{Behavioral experiments}

Flies were transferred to fresh food vials for up to $48 \mathrm{~h}$ before testing. Behavioral experiments were performed in dim red light at $80 \%$ relative humidity using 3 -octanol (1/100 dilution in mineral oil presented in a cup of $14-\mathrm{mm}$ diameter) and 4-methylcyclohexanol (1/150 dilution in mineral oil presented in a cup of 14-mm diameter) as olfactory stimuli. The US was represented either by a train of $12 \mathrm{DC}$ pulses of $120 \mathrm{~V}$ administered in rapid succession with 5-sec intervals as originally introduced by Tully and Quinn (1985) or by various numbers of DC pulses as indicated. To measure their performance, flies were always tested exactly 3 min after onset of training, except for flies that had received 24 pulses. Cohorts that expressed RNAi were raised and kept at $30^{\circ} \mathrm{C}$ to boost transgene expression.

\section{Generation of transgenic flies}

To construct the upstream activating sequence (UAS)-expression vectors containing wild-type epac cDNA, we obtained a full-length cDNA clone GH01501 containing the epac RD-isoform from the Drosophila Genomics Resource Center (DGRC). cDNA was PCR amplified and cloned into the pEntry vector according to the manufacturer's protocol (pENTR/D-TOPO Cloning Kit, Invitrogen Inc.) and further cloned into the pUAST Drosophila transfection vector obtained from DGRC. Generation of transgenic Drosophila by germ-line transformation was performed by BestGene Inc.

\section{Generation of small deletions covering the Drosophila epac locus}

We used the Drosophila FRT-derived deletion (FDD) system (Parks et al. 2004) to generate loss-of-function alleles for the unique
Drosophila epac gene. Drosophila epac is located on the right arm of chromosome 2 and was partially deleted upon FRT dependent remobilization of trans heterozygote P-element combinations. Deletions were verified by PCR and subsequently sequenced.

\section{PKA assay}

PKA activity was determined in head homogenate of 7-d-old adult females expressing UAS-DCO-RNAi under control of the neuron-specific elav-Gal4 element. PKA activity was determined using a phosphorylation assay following the manufacturer's procedures (PepTag Non-Radioactive cAMP dependent Protein Kinase Assay System; Promega).

\section{Competing interest statement}

The authors declare no competing financial interests.

\section{Acknowledgments}

We are thankful to Anne Carney for thorough editing of the manuscript. Fly stocks were obtained from the Bloomington Stock Center (Indiana, USA) and from VDRC (Vienna, Austria). This work was supported by DFG grant SCHW1410/1-1 to M.S.

\section{References}

Aso Y, Siwanowicz I, Bracker L, Ito K, Kitamoto T, Tanimoto H. 2010. Specific dopaminergic neurons for the formation of labile aversive memory. Curr Biol 20: 1445-1451.

Aso Y, Hattori D, Yu Y, Johnston RM, Iyer NA, Ngo TT, Dionne H, Abbott LF, Axel R, Tanimoto H, et al. 2014. The neuronal architecture of the mushroom body provides a logic for associative learning. Elife 3: e04577.

Barnstedt O, Owald D, Felsenberg J, Brain R, Moszynski JP, Talbot CB, Perrat PN, Waddell S. 2016. Memory-relevant mushroom body output synapses are cholinergic. Neuron 89: 1237-1247.

Benarroch EE. 2013. HCN channels: function and clinical implications. Neurology 80: 304-310.

Bertinetti D, Schweinsberg S, Hanke SE, Schwede F, Bertinetti O, Drewianka S, Genieser HG, Herberg FW. 2009. Chemical tools selectively target components of the PKA system. BMC Chem Biol 9: 3.

Bos JL. 2006. Epac proteins: multi-purpose cAMP targets. Trends Biochem Sci 31: $680-686$.

Boto T, Louis T, Jindachomthong K, Jalink K, Tomchik SM. 2014. Dopaminergic modulation of cAMP drives nonlinear plasticity across the Drosophila mushroom body lobes. Curr Biol 24: 822-831.

Bouzaiane E, Trannoy S, Scheunemann L, Placais PY, Preat T. 2015. Two independent mushroom body output circuits retrieve the six discrete components of Drosophila aversive memory. Cell Rep 11: 1280-1292. 
Chen H, Tsalkova T, Chepurny OG, Mei FC, Holz GG, Cheng X, Zhou J. 2013. Identification and characterization of small molecules as potent and specific EPAC2 antagonists. I Med Chem 56: 952-962.

Cooper DM, Tabbasum VG. 2014. Adenylate cyclase-centred microdomains. Biochem J 462: 199-213.

DasGupta S, Ferreira CH, Miesenbock G. 2014. FoxP influences the speed and accuracy of a perceptual decision in Drosophila. Science 344: 901-904.

de Rooij J, Zwartkruis FJ, Verheijen MH, Cool RH, Nijman SM, Wittinghofer A, Bos JL. 1998. Epac is a Rap1 guanine-nucleotide-exchange factor directly activated by cyclic AMP. Nature 396: 474-477.

Efetova M, Petereit L, Rosiewicz K, Overend G, Haussig F, Hovemann BT, Cabrero P, Dow JA, Schwarzel M. 2013. Separate roles of PKA and EPAC in renal function unraveled by the optogenetic control of cAMP levels in vivo. J Cell Sci 126: 778-788.

Enserink JM, Christensen AE, de Rooij J, van Triest M, Schwede F, Genieser HG, Doskeland SO, Blank JL, Bos JL. 2002. A novel Epac-specific cAMP analogue demonstrates independent regulation of Rap1 and ERK. Nat Cell Biol 4: 901-906.

Gelinas JN, Banko JL, Peters MM, Klann E, Weeber EJ, Nguyen PV. 2008. Activation of exchange protein activated by cyclic-AMP enhances long-lasting synaptic potentiation in the hippocampus. Learn Mem 15: 403-411.

Gloerich M, Bos JL. 2010. Epac: defining a new mechanism for cAMP action. Annu Rev Pharmacol Toxicol 50: 355-375.

Guo A, Li L, Xia SZ, Feng CH, Wolf R, Heisenberg M. 1996. Conditioned visual flight orientation in Drosophila: dependence on age, practice, and diet. Learn Mem 3: 49-59.

He C, Chen F, Li B, Hu Z. 2014. Neurophysiology of HCN channels: from cellular functions to multiple regulations. Prog Neurobiol 112: 1-23.

Heisenberg M. 2003. Mushroom body memoir: from maps to models. Nat Rev Neurosci 4: 266-275.

Hige T, Aso Y, Modi MN, Rubin GM, Turner GC. 2015. Heterosynaptic plasticity underlies aversive olfactory learning in Drosophila. Neuron 88: 985-998.

Kandel ER. 2001. The molecular biology of memory storage: a dialog between genes and synapses. Biosci Rep 21: 565-611.

Kawasaki H, Springett GM, Mochizuki N, Toki S, Nakaya M, Matsuda M, Housman DE, Graybiel AM. 1998. A family of cAMP-binding proteins that directly activate Rap1. Science 282: 2275-2279.

Lane ME, Kalderon D. 1993. Genetic investigation of cAMP-dependent protein kinase function in Drosophila development. Genes Dev 7: $1229-1243$.
Laurent AC, Breckler M, Berthouze M, Lezoualc'h F. 2012. Role of Epac in brain and heart. Biochem Soc Transac 40: 51-57.

Ma N, Abel T, Hernandez PJ. 2009. Exchange protein activated by cAMP enhances long-term memory formation independent of protein kinase A. Learn Mem 16: 367-370.

McGuire SE, Le PT, Osborn AJ, Matsumoto K, Davis RL. 2003. Spatiotemporal rescue of memory dysfunction in Drosophila. Science 302: $1765-1768$.

Moressis A, Friedrich AR, Pavlopoulos E, Davis RL, Skoulakis EM. 2009. A dual role for the adaptor protein DRK in Drosophila olfactory learning and memory. J Neurosci 29: 2611-2625.

Owald D, Waddell S. 2015. Olfactory learning skews mushroom body output pathways to steer behavioral choice in Drosophila. Curr Opin Neurobiol 35: 178-184

Parks AL, Cook KR, Belvin M, Dompe NA, Fawcett R, Huppert K, Tan LR, Winter CG, Bogart KP, Deal JE, et al. 2004. Systematic generation of high-resolution deletion coverage of the Drosophila melanogaster genome. Nat Genet 36: 288-292.

Rocher A, Caceres AI, Almaraz L, Gonzalez C. 2009. EPAC signalling pathways are involved in low $\mathrm{PO} 2$ chemoreception in carotid body chemoreceptor cells. J Physiol 587: 4015-4027.

Sakaba T, Neher E. 2003. Involvement of actin polymerization in vesicle recruitment at the calyx of Held synapse. J Neurosci 23: 837-846.

Schmidt M, Dekker FJ, Maarsingh H. 2013. Exchange protein directly activated by cAMP (epac): a multidomain cAMP mediator in the regulation of diverse biological functions. Pharmacol Rev 65: 670-709.

Ster J, De Bock F, Guerineau NC, Janossy A, Barrere-Lemaire S, Bos JL, Bockaert J, Fagni L. 2007. Exchange protein activated by cAMP (Epac) mediates cAMP activation of p38 MAPK and modulation of $\mathrm{Ca}^{2+}$-dependent $\mathrm{K}^{+}$channels in cerebellar neurons. Proc Natl Acad Sci 104: $2519-2524$

Tully T, Quinn WG. 1985. Classical conditioning and retention in normal and mutant Drosophila melanogaster. J Comp Physiol [A] 157: 263-277.

Yang Y, Shu X, Liu D, Shang Y, Wu Y, Pei L, Xu X, Tian Q, Zhang J, Qian K, et al. 2012. EPAC null mutation impairs learning and social interactions via aberrant regulation of miR-124 and Zif268 translation. Neuron 73: $774-788$.

Zhong N, Zucker RS. 2005. cAMP acts on exchange protein activated by cAMP/cAMP-regulated guanine nucleotide exchange protein to regulate transmitter release at the crayfish neuromuscular junction. $J$ Neurosci 25: 208-214.

Received September 12, 2016; accepted in revised form March 10, 2017. 


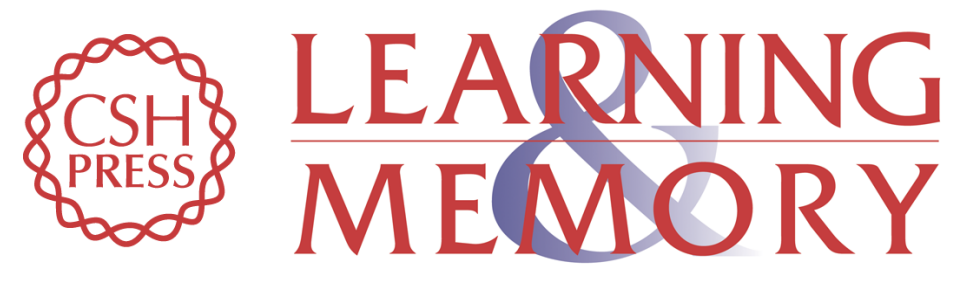

\section{Null EPAC mutants reveal a sequential order of versatile cAMP effects during Drosophila aversive odor learning}

Antje Richlitzki, Philipp Latour and Martin Schwärzel

Learn. Mem. 2017, 24:

Access the most recent version at doi:10.1101/Im.043646.116

\begin{aligned} & \hline References $\begin{array}{l}\text { This article cites } 36 \text { articles, } 16 \text { of which can be accessed free at: } \\ \text { http://learnmem.cshlp.org/content/24/5/210.full.html\#ref-list-1 }\end{array} \\ & \begin{array}{r}\text { Creative } \\ \text { Commons } \\ \text { License }\end{array} \begin{array}{l}\text { This article is distributed exclusively by Cold Spring Harbor Laboratory Press for the } \\ \text { first } 12 \text { months after the full-issue publication date (see } \\ \text { http://learnmem.cshlp.org/site/misc/terms.xhtml). After } 12 \text { months, it is available under } \\ \text { a Creative Commons License (Attribution-NonCommercial } 4.0 \text { International), as } \\ \text { described at http://creativecommons.org/licenses/by-nc/4.0/. }\end{array} \\ & \begin{array}{c}\text { Receive free email alerts when new articles cite this article - sign up in the box at the } \\ \text { top right corner of the article or click here. }\end{array} \\ & \begin{array}{l}\text { Service } \\ \text { terting }\end{array}\end{aligned}$ 